\title{
ON AUTOMORPHISMS OF STRONGLY REGULAR GRAPHS WITH $\lambda=0$ AND $\mu=3$
}

\author{
A. A. MAKHNEV AND V. V. NOSOV
}

\begin{abstract}
A strongly regular graph with $\lambda=0$ and $\mu=3$ is of degree 3 or 21 . The automorphisms of prime order and the subgraphs of their fixed points are described for a strongly regular graph $\Gamma$ with parameters $(162,21,0,3)$. In particular, the inequality $|G / O(G)| \leq 2$ holds true for $G=\operatorname{Aut}(\Gamma)$.
\end{abstract}

\section{INTRODUCTION}

We consider unoriented graphs without loops and multiple edges. If $a$ and $b$ are vertices of a graph $\Gamma$, then $d(a, b)$ denotes the distance between $a$ and $b$, and $\Gamma_{i}(a)$ stands for the subgraph of $\Gamma$ that is induced by the set of vertices that are at the distance $i$ in $\Gamma$ from the vertex $a$. The subgraph $\Gamma_{1}(a)$ is called the neighborhood of $a$ and is denoted by $[a]$. By $a^{\perp}$ we denote the subgraph that is the ball of radius 1 with center $a$.

A graph $\Gamma$ is said to be regular of degree $k$ if $[a]$ contains precisely $k$ vertices for any vertex $a$ in $\Gamma$. A graph $\Gamma$ is edge-regular with parameters $(v, k, \lambda)$ if $\Gamma$ contains $v$ vertices, is regular of degree $k$, and every edge in $\Gamma$ lies in $\lambda$ triangles. A graph $\Gamma$ is completely regular with parameters $(v, k, \lambda, \mu)$ if $\Gamma$ is edge-regular with the respective parameters and the subgraph $[a] \cap[b]$ contains $\mu$ vertices in the case where $d(a, b)=2$. A completely regular graph of diameter 2 is said to be strongly regular. We denote by $K_{m_{1}, \ldots, m_{n}}$ the complete $n$-partite graph with parts of orders $m_{1}, \ldots, m_{n}$. If $m_{1}=\cdots=m_{n}=m$, then this graph is denoted by $K_{n \times m}$. The graph $K_{1,3}$ is called a 3 -paw. For a subgraph $\Delta$ of a graph $\Gamma$, we denote by $X_{i}(\Delta)$ the set of all vertices in $\Gamma-\Delta$ that are adjacent with precisely $i$ vertices in $\Delta$, and we put $x_{i}(\Delta)=\left|X_{i}(\Delta)\right|$.

For a subset $X$ in the automorphism group of a graph $\Gamma$, we let $\operatorname{Fix}(X)$ denote the set of all vertices in $\Gamma$ that are fixed under the action of any automorphism in $X$.

The subgraphs of fixed points for automorphisms of a strongly regular graph with small values of the parameters $\lambda$ and $\mu$ have a rigidly determined structure. For example, the subgraph of fixed points of an automorphism of a Moore graph is itself a Moore graph or a star (see 1, Lemma 1]). The automorphisms of strongly regular graphs with $\lambda=0$ and $\mu=2$ were considered in [2].

In the present paper, we study the subgraphs of fixed points for automorphisms of strongly regular graphs with $(\lambda, \mu)=(0,3)$.

Proposition. Let $\Gamma$ be a strongly regular graph with $\lambda=0$ and $\mu=3$. Then $\Gamma$ has the parameters $(6,3,0,3)$ or $(162,21,0,3)$.

Proof. Consider a strongly regular graph $\Gamma$ with parameters $(v, k, 0,3)$. Using the relation $(\lambda-\mu)^{2}+4(k-\mu)=n^{2}$, we obtain $n^{2}=4 k-3$; therefore, $n=2 u+1, k=u^{2}+u+1$,

2010 Mathematics Subject Classification. Primary 05C60, 05C69.

Key words and phrases. Edge-regular graph, completely regular graph, strongly regular graph, paw, clique.

Supported by RFBR (grants nos. 08-01-00009 and 08-01-90006). 
$n-m=u-1$, and $-m=-u-2$. From the rectangular relation $k(k-\lambda-1)=\mu(v-k-1)$, we find $v=\left(u^{4}+2 u^{3}+5 u^{2}+4 u+6\right) / 3$. By the integrality condition, the multiplicity of the positive nonprincipal eigenvalue of the graph $\Gamma$ is equal to $f=(m-1) k(k+m) /(n \mu)=$ $(u+1)\left(u^{2}+u+1\right)\left(u^{2}+2 u+3\right) /(6 u+3)$. Hence, $2 u+1$ divides 9 , so that $u=1$ or 4. For $u=1$, we obtain the graph $K_{3,3}$, but for $u=4$ the graph $\Gamma$ has the parameters $(162,21,0,3)$. In the latter case, $\Gamma$ has nonprincipal eigenvalues 3 and -6 of multiplicities 105 and 56, respectively. The proposition is proved.

We note that a strongly regular graph $\Gamma$ with parameters $(162,21,0,3)$ satisfies all known necessary existence conditions for strongly regular graphs. Next, in view of the Hoffman bound, the number of vertices in any coclique of $\Gamma$ does not exceed 36 .

Theorem. Suppose $\Gamma$ is a strongly regular graph with parameters $(162,21,0,3), G=$ $\operatorname{Aut}(\Gamma), g$ is an element of prime order $p$ in $G, \Omega=\operatorname{Fix}(g), X_{i}=X_{i}(\Omega)$, and $x_{i}=\left|X_{i}\right|$. Then one of the following statements is valid:

(1) $p=2$ and either $|\Omega|=0$ or $\Omega$ is a 3-paw;

(2) $p=3$ and either

(i) $\Omega$ is a $K_{3,3}$-subgraph, $x_{1}=108, x_{3}=0, x_{0}=48$, a vertex of $X_{0}$ is adjacent to 18 vertices of $X_{1}$ and to 3 vertices of $X_{0}$, and a vertex of $X_{1}$ is adjacent to 12 vertices of $X_{1}$ and to 8 vertices of $X_{0}$; or

(ii) $\Omega$ is the union of three pairwise isolated $K_{3,3}$-subgraphs, $x_{3}=108, x_{0}=36$, the neighborhood of every vertex in $X_{0}$ contains 18 vertices of $X_{3}$ and 3 vertices of $X_{0}$, and the neighborhood of every vertex in $X_{3}$ contains 12 vertices of $X_{3}$ and 6 vertices of $X_{0}$; or

(iii) $\Omega$ has three isolated vertices $u_{1}, u_{2}, u_{3}$ and two $K_{3,3}$-components $\Omega^{1}$ and $\Omega^{2}$; moreover, $u_{1}, u_{2}, u_{3}$ lie in a $K_{3,3}$-subgraph of $X_{0}\left(\Omega^{1} \cup \Omega^{2}\right), x_{0}=36, x_{1}=0, x_{2}=54$, and $x_{3}=57$; or

(iv) $\Omega$ contains three isolated vertices $u_{1}, u_{2}, u_{3}$ and a $K_{3,3}$-component $\Omega^{1}$; moreover, $u_{1}, u_{2}, u_{3}$ lie in a $K_{3,3}$-subgraph of $X_{0}\left(\Omega^{1}\right)$, and $x_{3}=3, x_{2}=54, x_{1}=54, x_{0}=42$; or

(v) $\Omega$ is a $3 \alpha$-coclique, $\alpha \leq 5$;

(3) $p=5$ and $\Omega$ is a two-vertex clique;

(4) $p=7$ and $\Omega$ is a one-vertex clique.

The theorem implies that $|G / O(G)| \leq 2$. Indeed, if $G$ contains an involution $t$, then, by the theorem, $t$ is an odd permutation on $\Omega$; therefore, $G=O(G)\langle t\rangle$.

In $\S 1$ we present some auxiliary results, and $\S \S 2$ and 3 are devoted to the proof of the theorem.

\section{§1. Preliminary Results}

In this section, we present auxiliary results that are used in the proof of the theorem. The following lemma (see [3]) is an analog of the Hoffman bound.

Lemma 1.1. Let $\Gamma$ be a strongly regular graph on $v$ vertices with eigenvalues $k, r, s$, and let $\Omega$ be a regular subgraph of $\Gamma$ of degree $k^{\prime}$ on $u$ vertices, $u<v$. Then $s \leq$ $k^{\prime}-u\left(k-k^{\prime}\right) /(v-u) \leq r$; moreover, if equality is attained in one of these nonstrict inequalities, then each vertex in $\Gamma-\Omega$ is adjacent to precisely $u\left(k-k^{\prime}\right) /(v-u)$ vertices in $\Omega$.

Lemma 1.2 (Bose-Dowling). Let $\Gamma$ be a connected graph in which $|[a] \cap[b]|=\lambda$ for any adjacent vertices $a$ and $b$ and $|[u] \cap[w]|=\mu$ for any vertices $u$ and $w$ that are at distance 2 . Then either $\Gamma$ is a regular graph, or $\mu=1$.

Proof. This is Proposition 1.4.1 in 4. 
Higman's method for handling automorphisms of a strongly regular graph is given in Cameron's monograph [5, Chapter 3]. Moreover, the graph $\Gamma$ is regarded as a symmetric association scheme $\left(X,\left\{R_{0}, R_{1}, R_{2}\right\}\right)$, where $X$ is the set of vertices of the graph, $R_{0}$ is the equality relation on $X, R_{1}$ is the adjacency relation in $\Gamma$, and $R_{2}$ is the adjacency relation in the complementary graph $\bar{\Gamma}$. If $P$ and $Q$ are the first and the second matrix of eigenvalues of the scheme, then

$$
P=\left(\begin{array}{ccc}
1 & 1 & 1 \\
k & r & s \\
v-k-1 & -r-1 & -s-1
\end{array}\right)
$$

and $P Q=Q P=v I$. Here $v$ is the number of vertices, and $k, r$, and $s$ are the eigenvalues of the graph $\Gamma$ with multiplicities $1, f$, and $g$, respectively (these multiplicities form the first column of the matrix $Q$ ).

The permutational representation of the group $G=\operatorname{Aut}(\Gamma)$ on the vertices of the graph $\Gamma$ gives rise to a matrix representation $\psi$ of the group $G$ in $G L(v, \mathbf{C})$ in the usual way. Let $\chi_{i}$ be the character of the representation of $G$ obtained by projecting $\psi$ to the $i$ th eigenspace $W_{i}$ of the adjacency matrix of $\Gamma, i=0,1,2$. Then for any $g \in \operatorname{Aut}(\Gamma)$ we get

$$
\chi_{i}(g)=v^{-1} \sum_{j=0}^{2} Q_{i j} \alpha_{j}(g),
$$

where $\alpha_{0}(g)=|\operatorname{Fix}(g)|, \alpha_{1}(g)$ is the number of points $x$ in $X$ such that $x$ and $x^{g}$ are adjacent in $\Gamma$, and $\alpha_{2}(g)$ is the number of points $x$ in $X$ such that $x$ and $x^{g}$ are adjacent in the complementary graph $\bar{\Gamma}$.

We note that the values of the characters are integral algebraic numbers, and if the right-hand side of the expression for $\chi_{i}(g)$ is a rational number, then $\chi_{i}(g)$ is an integer.

\section{§2. Automorphisms Without FiXed POINTS AND AUTOMORPHISMS OF ODD ORDER}

Let $\Gamma$ be a strongly regular graph with parameters $(162,21,0,3)$, and let $G=\operatorname{Aut}(\Gamma)$. Then $\Gamma$ has eigenvalues $21,3,-6$, and

$$
P=\left(\begin{array}{ccc}
1 & 1 & 1 \\
21 & -6 & 3 \\
140 & 5 & -4
\end{array}\right), \quad Q=\left(\begin{array}{ccc}
1 & 1 & 1 \\
56 & -16 & 2 \\
105 & 15 & -3
\end{array}\right)
$$

Therefore, the value of the character obtained by projecting to the subspace of dimension 56 is equal to

$$
\chi_{1}(g)=\left(56 \alpha_{0}(g)-16 \alpha_{1}(g)+2 \alpha_{2}(g)\right) / 162 .
$$

Substituting $\alpha_{2}(g)=162-\alpha_{0}(g)-\alpha_{1}(g)$ in this formula, we obtain

$$
\chi_{1}(g)=\left(3 \alpha_{0}(g)-\alpha_{1}(g)\right) / 9+2 .
$$

Lemma 2.1. If $\Delta$ is a regular subgraph in $\Gamma$ of degree $k^{\prime}$ with $u$ vertices, then:

(1) $-6 \leq k^{\prime}-u\left(21-k^{\prime}\right) /(162-u) \leq 3$;

(2) if $k^{\prime}=6$, then $27 \leq u \leq 72$;

(3) if $k^{\prime}=12$, then $81 \leq u \leq 108$.

Proof. By Lemma 1.1, we have $-6 \leq k^{\prime}-u\left(21-k^{\prime}\right) /(162-u) \leq 3$.

If $k^{\prime}=6$, then $-6 \leq 6-15 u /(162-u) \leq 3$ and $27 \leq u \leq 72$.

If $k^{\prime}=12$, then $-6 \leq 12-3 u /(162-u) \leq 3$ and $81 \leq u \leq 108$.

Lemma 2.2. If an element $g \in G$ of prime order $p$ acts without fixed points on $\Gamma$, then $p=2$, any vertex $a \in \Gamma$ is adjacent to $a^{g}$, the set of vertices of the graph $\Gamma$ is partitioned by the $g$-admissible edges, and the graph $\Gamma^{*}$ on the set of the $g$-admissible edges in which 
two edges are adjacent if a vertex in one of them is adjacent to a vertex in the other is strongly regular with parameters $(81,20,1,6)$.

Proof. Let $g$ be an element of prime order in $G$ that acts without fixed points on $\Gamma$. Since $v=162$, we have $p=2$ or $p=3$.

Let $p=2$. Then any vertex $a \in \Gamma$ is adjacent to $a^{g}$; otherwise, the subgraph $[a] \cap\left[a^{g}\right]$ contains a vertex belonging to $\operatorname{Fix}(g)$. Next, the set of vertices of $\Gamma$ is partitioned by $g$-admissible edges. We define a graph $\Gamma^{*}$ on the set of $g$-admissible edges, assuming that two such edges are adjacent if a vertex in one of them is adjacent to a vertex in the other. Then $\Gamma^{*}$ is a regular graph of degree 20. If an edge $\left\{a, a^{g}\right\}$ is adjacent to an edge $\left\{b, b^{g}\right\}$, and, say, $a$ is adjacent to $b$, then $[a] \cap\left[b^{g}\right]$ contains $a^{g}, b$, and yet another vertex. Therefore, $\Gamma^{*}$ is an edge-regular graph with $\lambda^{*}=1$. If the edge $\left\{a, a^{g}\right\}$ is not adjacent to the edge $\left\{b, b^{g}\right\}$, then each of the subgraphs $[a] \cap[b]$ and $[a] \cap\left[b^{g}\right]$ contains 3 vertices, so that $\Gamma^{*}$ is a coedge-regular graph with $\mu^{*}=6$. Therefore, if $p=2$, the claim follows.

Let $p=3$. Then no vertex $a \in \Gamma$ is adjacent to $a^{g}$; otherwise, the vertices $a, a^{g}$, and $a^{g^{2}}$ form a triangle. Thus, for $b \in[a] \cap\left[a^{g}\right]$ the subgraph $\Delta=\left\{a, a^{g}, a^{g^{2}} ; b, b^{g}, b^{g^{2}}\right\}$ is a hexagon or a $K_{3,3}$-subgraph. We define a graph $\Gamma^{\prime}$ on the set of $\langle g\rangle$-orbits, assuming that two orbits are adjacent if a vertex in one of them is adjacent to a vertex in the other. If $\Delta$ is a $K_{3,3}$-subgraph, then $a$ is adjacent to 18 vertices off $\Delta$, and the degree of $\left\{a, a^{g}, a^{g^{2}}\right\}$ in the graph $\Gamma^{\prime}$ is equal to 19. If $\Delta$ is a hexagon, then $a$ is adjacent to pairs of vertices in three $\langle g\rangle$-orbits, and the degree of $\left\{a, a^{g}, a^{g^{2}}\right\}$ in $\Gamma^{\prime}$ is equal to 18 . We denote by $X$ the orbit $\left\{x, x^{g}, x^{g^{2}}\right\}$, and by $\Gamma_{i}^{\prime}(X)$ the $i$ th neighborhood of the vertex $X$ in the graph $\Gamma^{\prime}$. If $A$ is an orbit of degree 19 , then we denote by $A^{*}$ the orbit each vertex of which is adjacent to all vertices of $A$.

We show that $\Gamma^{\prime}$ contains no vertices of degree 19. Let $A$ be a vertex of degree 19 . Clearly, $\lambda^{\prime}\left(A, A^{*}\right)=0$. If $A$ and $C$ are adjacent orbits, and, in $C$, the vertex $a$ is adjacent only to $c$, then each of the subgraphs $[c] \cap\left[a^{g}\right]$ and $[c] \cap\left[a^{g^{2}}\right]$ contains 3 vertices that do not lie in $A^{*}$, whence $\lambda^{\prime}(A, C)=6$. Let $B \in \Gamma_{2}^{\prime}(A)$. If $b$ is adjacent to a vertex in $A^{*}$, then $\mu^{\prime}(A, B)=7$; otherwise, $\mu^{\prime}(A, B)=9$. Let $\Gamma^{\prime}(A)$ contain $1+\sigma$ vertices of degree 19 , and let $\Gamma_{2}^{\prime}(A)$ contain $\delta_{i}$ vertices adjacent to precisely $i$ vertices in $\Gamma^{\prime}(A)$. Then $\delta_{7}+\delta_{9}=34$ and $7 \delta_{7}+9 \delta_{9}=18+12 \sigma+11(18-\sigma)=216+\sigma, \sigma \leq 19$, which contradicts the relation $7 \delta_{7}+7 \delta_{9}=238$.

Therefore, $\Gamma^{\prime}$ is a regular graph of degree 18. An edge $\{A, C\}$ is said to be thick (thin) if the vertex $a$ is adjacent to two vertices (one vertex) in $\left\{c, c^{g}, c^{g^{2}}\right\}$. Let $\{A, C\}$ be an edge, and let $B \in \Gamma^{\prime}(A) \cap \Gamma^{\prime}(C)$.

First, we observe that the triangle $\{A, B, C\}$ cannot contain two thick edges. Suppose the edges $\{A, C\}$ and $\{A, B\}$ are thick. Then $a$ is adjacent to vertices $b_{1}$ and $b_{2}$ in $B$ and to vertices $c_{1}$ and $c_{2}$ in $C$, which contradicts the fact that either $b_{1}$ or $b_{2}$ is adjacent to a vertex of $\left\{c_{1}, c_{2}\right\}$. Let the edge $\{A, C\}$ be thick, and let $a$ be adjacent to a vertex $b$ in $B$ and to vertices $c$ and $c^{g}$ in $C$. Then $c^{g}$ is adjacent to $a, a^{g}$, and $b^{g^{2}}$. Next, since $\left[c^{g^{2}}\right] \cap[a]$ contains 3 vertices, we have $\lambda^{\prime}(A, C)=3$.

Suppose the edge $\{A, C\}$ is thin (and $a$ is adjacent to $c$ ), and the edge $\{A, B\}$ is thick (and $a$ is adjacent to $b$ and $b^{g}$ ). If $D \in \Gamma^{\prime}(A) \cap \Gamma^{\prime}(C)$ and the edge $\{A, D\}$ is thick $(a$ is adjacent to $d$ and $\left.d^{g}\right)$, then $c$ is adjacent to $b^{g^{2}}$ and $d^{g^{2}}$, and in particular, $\left[a^{g}\right] \cap[c]$ contains $b^{g^{2}}$ and $d^{g^{2}}$. If $D \in \Gamma^{\prime}(A) \cap \Gamma^{\prime}(C)$ and the edge $\{C, D\}$ is thick ( $c$ is adjacent to $d$ and $\left.d^{g}\right)$, then $a^{g}$ and $a^{g^{2}}$ are adjacent to $b^{g^{2}}, a$ is adjacent to $d^{g^{2}}$, and $\left[a^{g}\right] \cap[c]$ contains $b^{g^{2}}$ and a vertex of $\left\{d, d^{g}\right\}$.

Since each of the subgraphs $\left[a^{g}\right] \cap[c]$ and $\left[a^{g^{2}}\right] \cap[c]$ contains 3 vertices, it follows that $\lambda^{\prime}(A, C) \leq 6$, and moreover, if $\lambda^{\prime}(A, C)<6$, then some orbit contains a vertex adjacent 
to $a^{g}, a^{g^{2}}$, and $c$. But in this case the subgraphs $\left[c^{g}\right] \cap[a]$ and $\left[c^{g^{2}}\right] \cap[a]$ also intersect; therefore, some orbit contains a vertex adjacent to $c^{g}, c^{g^{2}}$, and $a$, and $\lambda^{\prime}(A, C) \leq 4$. If there are two orbits that contain vertices adjacent to $a^{g}, a^{g^{2}}$, and $c$, then there are two orbits that contain vertices adjacent to $c^{g}, c^{g^{2}}$, and $a$; this contradicts the fact that $\left|\left[a^{g}\right] \cap[c]\right| \geq 4$.

Thus, $\lambda^{\prime}(A, C)=3$ if the edge $\{A, C\}$ is thick, and $\lambda^{\prime}(A, C)$ is 4 or 6 if the edge $\{A, C\}$ is thin.

Let $\{A, B\}$ be vertices that are at distance 2 in $\Gamma^{\prime}$ and $D \in \Gamma^{\prime}(A) \cap \Gamma^{\prime}(B)$. Since $[a] \cap[b]$ contains 3 vertices for $b \in B$, we have $\mu^{\prime}(A, B) \leq 9$; moreover, if $\mu^{\prime}(A, B)<9$, then some orbit $C$ contains a vertex adjacent to $a, b$, and $b^{g}$ for an appropriate vertex $b \in B$. But in this case, some orbit $D$ contains a vertex adjacent to a vertex in $B$ and to two vertices in $A$, whence $\mu^{\prime}(A, B) \leq 7$. Also, for $a \in A$ there are two pairs of vertices in $B$ such that every vertex in the first pair is adjacent to a vertex in $[a] \cap C$, and every vertex in the second pair is adjacent to a vertex in $[a] \cap D$. Thus, $\mu^{\prime}(A, B)$ equals 5,7 , or 9.

The subgraph $\Gamma^{\prime}(A)$ contains precisely three vertices $C_{1}, C_{2}$, and $C_{3}$ connected with $A$ by thick edges, and the number of thick edges between $\Gamma_{2}^{\prime}(A)$ and $C_{1}, C_{2}$, and $C_{3}$ is equal to 6 . Since every vertex $B$ with $\mu^{\prime}(A, B)=5$ is connected by two thick edges with vertices in $\left\{C_{1}, C_{2}, C_{3}\right\}$, the number of such vertices does not exceed 3 .

Now the number of edges between $\Gamma^{\prime}(A)$ and $\Gamma_{2}^{\prime}(A)$ is at most $3 \cdot 14+15 \cdot 13=237$ and at least $3 \cdot 5+32 \cdot 7=239$, a contradiction. The lemma is proved.

We fix an element $g$ of prime order $p$ in $G$ such that $\Omega=\operatorname{Fix}(g)$ is a nonempty graph. Let $X_{i}$ be the set of vertices in $\Gamma-\Omega$ that are adjacent to precisely $i$ vertices in $\Omega$, and let $x_{i}=\left|X_{i}\right|$.

Lemma 2.3. If $p>3$, then either

(1) $p=7, \Omega$ is a one-vertex clique, and $\alpha_{1}(g)=21$; or

(2) $p=5, \Omega$ is a two-vertex clique, $\alpha_{1}(g)=15$ or 60 , and $\alpha_{1}(g)+\alpha_{1}\left(g^{2}\right)<120$.

Proof. If $p>3$ and $\Omega$ is not a clique, then for any two nonadjacent vertices $a, b \in \Omega$, the subgraph $[a] \cap[b]$ is $g$-admissible and is contained in $\Omega$. Therefore, $\Omega$ is a clique or a strongly regular graph with $\lambda=0$ and $\mu=3$. In the latter case, $\Omega$ is a $K_{3,3}$-subgraph and $|\Gamma-\Omega|=156$. Then $x_{0}=48$ and $x_{1}=108$, which contradicts the fact that $g$ acts without fixed points on $X_{0}$.

If $p>3$ and $\Omega$ is a clique, then either $|\Omega|=1$ and $p$ divides 21 and 161, or $|\Omega|=2$ and $p=5$. In the first case, we have $p=7$, every $\langle g\rangle$-orbit is a coclique or a heptagon, $\chi_{1}(g)=\left(3-\alpha_{1}(g)\right) / 9+2$, and $\alpha_{1}(g)-3$ is divisible by 9 . Therefore, $\alpha_{1}(g)$ is equal to 21 or 84 . Let the vertices $a$ and $a^{g}$ be adjacent, and let a vertex $b$ in the heptagonal $\langle g\rangle$-orbit be adjacent to two vertices in $a^{\langle g\rangle}$. Then either $b$ is adjacent to vertices that are at distance 2 in $a^{\langle g\rangle}$ and $b$ is adjacent to $b^{g^{3}}$, or $b$ is adjacent to vertices that are at distance 3 in $a^{\langle g\rangle}$ and $b$ is adjacent to $b^{g^{2}}$. This implies that if a vertex is adjacent to three vertices in $a^{\langle g\rangle}$, then it lies in a coclique $\langle g\rangle$-orbit and such an orbit is unique.

Suppose $\alpha_{1}(g)=84$. Then $\alpha_{1}\left(g^{2}\right)=\alpha_{1}\left(g^{3}\right)=21$, the number of heptagonal $\langle g\rangle$-orbits is equal to 18 , and the number of coclique $\langle g\rangle$-orbits of length 7 is equal to 5 (and three of these orbits lie in the neighborhood of a vertex $u$ fixed by $g$ ). On the set of $\langle g\rangle$-orbits of length 7, we define a graph $\Gamma^{\prime}$ by assuming that two orbits are adjacent if a vertex of one orbit is adjacent to a vertex of the other. We denote the orbit $\left\{x, x^{g}, \ldots, x^{g^{6}}\right\}$ by $X$. An edge $\{A, B\}$ of the graph $\Gamma^{\prime}$ is said to be $i$-thick if $a$ is adjacent to precisely $i$ in $B$, $i \geq 2$.

We say that a heptagonal $\langle g\rangle$-orbit $A$ is of type $(i)$ if $a$ is adjacent to $a^{g^{i}}$. Then an orbit of type (3) is connected by thick edges to at most three orbits of type (1), and 
an orbit of type (2) is connected by thick edges to at most two orbits of type (1) (thus, we obtain at most 15 thick edges between orbits of type (1) and orbits of types (2) and (3)). If there are no coclique orbits adjacent to triples of vertices in orbits of type (1), then the number of thick edges between orbits of type (1) and coclique orbits is at least $12(2+3)-15=45$. On the other hand, a coclique orbit outside the neighborhood of a fixed vertex is connected by thick edges with at most 9 orbits of type (1), and a coclique orbit in the neighborhood of a fixed vertex is connected by thick edges with at most 6 orbits of type (1); this contradicts the fact that the number of thick edges between orbits of type (1) and coclique orbits does not exceed $3 \cdot 6+2 \cdot 9=36$.

Suppose there are $\delta$ coclique orbits that contain vertices adjacent to triples of vertices in orbits of type (1). None of these $\delta$ orbits can contain a vertex adjacent to triples of vertices in different orbits of type (1). Therefore, the number of 2-thick edges between orbits of type (1) and coclique orbits is at least $(12-\delta)(2+3)-15=45-5 \delta$ and at most $(3 \cdot 6+2 \cdot 9)-3 \delta=36-3 \delta$. Hence, $\delta=5$, which contradicts the fact that the number of orbits of type (1) is equal to 12 . So, statement (1) is valid for $p=7$.

If $p=5$, then $\chi_{1}(g)=\left(6-\alpha_{1}(g)\right) / 9+2$ and $\alpha_{1}(g)-6$ is divisible by 9 . Thus, $\alpha_{1}(g)=15,60$ or 105 .

Suppose vertices $a$ and $a^{g}$ are adjacent. If a vertex $b$ in a pentagonal $\langle g\rangle$-orbit is adjacent to two vertices in $a^{\langle g\rangle}$, then we may assume that $a^{g}$ and $a^{g^{-1}}$ are adjacent to $b$. If $b$ is adjacent to $b^{g}$, then the vertices $b^{g}$ and $b^{g^{-1}}$ are adjacent to $a$, and $\mu(a, b) \geq 4$. If $b$ is adjacent to $b^{g^{2}}$, then $[a]$ contains the edge $\left\{b^{g}, b^{g^{-1}}\right\}$. In any case we arrive at a contradiction.

Suppose $\alpha_{1}(g)+\alpha_{1}\left(g^{2}\right)=120$. Then there are precisely 8 coclique $\langle g\rangle$-orbits of length 5 that lie in the neighborhood of vertices belonging to Fix $(g)$. On the set of $\langle g\rangle$-orbits of length 5 , we define a graph $\Lambda$ by assuming that two orbits are adjacent if a vertex of one orbit is adjacent to two vertices of the other. Then $\Lambda$ has two edges between a given pentagonal orbit and coclique orbits. Thus, the number of edges between the pentagonal and coclique orbits is equal to 48 , whence a certain coclique orbit $A$ is adjacent to at least 6 pentagonal orbits; this contradicts the fact that $A$ has 10 pairs of nonadjacent vertices that lie in two $\langle g\rangle$-orbits, and two nonadjacent vertices in $A$ belong to the neighborhood of three vertices from pentagonal orbits and one vertex from $\operatorname{Fix}(g)$.

Lemma 2.4. If $p=3$, then $\Omega$ is a subgraph as in the conclusion of the theorem.

Proof. Let $p=3$. Then $\Omega$ is not a clique (because 161 and 160 are not divisible by 3 ). Therefore, the connected components of the graph $\Omega$ are completely regular graphs with $\lambda=0$ and $\mu=3$; moreover, every vertex in $\Gamma-\Omega$ is adjacent to at most three vertices in $\Omega$. Let $X_{i}$ be the set of vertices in $\Gamma-\Omega$ that are adjacent to precisely $i$ vertices in $\Omega$, and let $x_{i}=\left|X_{i}\right|$. If $\Omega$ contains $t$ connected components $\Omega^{j}$ of degrees $k_{j}$, then $\sum x_{i}=|\Gamma-\Omega|, \sum i x_{i}=\sum_{j=1}^{t}\left(k-k_{j}\right)\left|\Omega^{j}\right|$, and $k_{j}$ is divisible by 3 .

Since every vertex in $\Gamma-\Omega$ is adjacent to at most three vertices in $\Omega$, and every vertex in $\Omega$ is adjacent to at least three vertices in $\Gamma-\Omega$, we have $|\Omega| \leq 81$. Therefore, $\Omega$ contains no vertices of degree greater than 12. If the degree of a vertex $a$ in $\Omega$ is equal to 12 , then $\left|\Omega_{2}(a)\right|=44$ and the number of edges between $\Omega$ and $\Gamma-\Omega$ is at least $57 \cdot 9$, which contradicts the inequality $|\Gamma-\Omega| \geq 57 \cdot 3$. If the degree of a vertex $a$ in $\Omega$ is 9 , then $\left|\Omega_{2}(a)\right|=24$ and the number of edges between $\Omega$ and $\Gamma-\Omega$ is at least $36 \cdot 12$, which is impossible because $|\Gamma-\Omega| \geq 36 \cdot 4$. Thus, $\Omega$ has no vertices of degree exceeding 6 .

We prove that if $\Omega$ contains a component $\Omega^{j}$ of degree 6 , then $\Omega=\Omega^{j}$. First, observe that $\left|\Omega_{2}^{j}(a)\right|=10$ and $\left|\Omega^{j}\right| \geq 18$ for $a \in \Omega^{j}$. If $b \in \Omega-\Omega^{j}$, then $[b]$ contains three vertices in each $\mu$-subgraph with vertices from $\Omega^{j}$ and $|[b]| \geq 18 \cdot 3 / 2$, a contradiction.

Let $\Omega$ be a connected graph of degree 6 and $|\Omega|=3 t$. Then for $a \in \Omega$ we obtain 
$\left|\Omega_{2}(a)\right|=10$. We show that $\Omega$ contains no $K_{3,3}$-subgraphs. Otherwise, for a vertex $a \in \Omega$ there are vertices $c, d \in \Omega_{2}(a)$ such that $[c] \cap \Omega(a)=[d] \cap \Omega(a)$. Hence, each of the eight vertices in $\Omega_{2}(a)-\{c, d\}$ is adjacent to at least two vertices in $\Omega(a)-[c]$, and a vertex in $\Omega(a)-[c]$ is adjacent to six vertices in $\Omega_{2}(a)$, a contradiction. Now, for $a \in \Omega$ and $d \in \Omega_{2}(a)$, the subgraph $\Omega_{2}(a)$ contains three vertices $c_{1}, c_{2}, c_{3}$ adjacent to pairs of vertices in $[d] \cap \Omega(a)$, and six vertices $e_{1}, \ldots, e_{6}$ each of which is adjacent to a unique vertex in $[d] \cap \Omega(a)$. In particular, $\Omega_{2}(a)$ is a coclique, $[d] \cap\left[c_{i}\right]$ contains a vertex from $\Omega_{3}(a)$, and $[d] \cap\left[e_{i}\right]$ contains two vertices from $\Omega_{3}(a)$. Therefore, every vertex in $\Omega_{3}(a)$ is adjacent to six vertices from $\Omega_{2}(a)$, in contradiction with $|\Omega|=22$.

Let $\Omega$ have $3 \alpha$ isolated vertices and $\beta$ components of degree 3 (which are obviously $K_{3,3}$-subgraphs). Then $\sum x_{i}=162-3 \alpha-6 \beta$ and $\sum i x_{i}=63 \alpha+18 \cdot 6 \beta$.

First, we assume that $\alpha=0$. If $|\Omega|=6$, then $x_{1}=108, x_{3}=0$, and $x_{0}=48$. For $z \in X_{0}$, the subgraph $[z]$ contains 18 vertices from $X_{1}$ and 3 vertices from $X_{0}$. If $w \in X_{1}$, then $[w]$ contains a vertex from $\Omega, 12$ vertices from $X_{1}$, and 8 vertices from $X_{0}$. In this case, statement (2)(i) of the theorem is valid.

If $\Omega$ is a disconnected graph with at least three components, then the number of edges between $\Omega$ and $\Gamma-\Omega$ is at least $18|\Omega|$; therefore $|\Omega|=18$ (otherwise $|\Gamma-\Omega| \geq 24 \cdot 18 / 3$ ). In this case, the neighborhood of every vertex in $\Omega$ contains 18 vertices from $X_{3}$, so that $x_{3}=108$ and $x_{0}=36$. Next, the neighborhood of every vertex in $X_{0}$ contains 18 vertices from $X_{3}$ and three vertices from $X_{0}$. Finally, the neighborhood of every vertex in $X_{3}$ contains 12 vertices from $X_{3}$ and 6 vertices from $X_{0}$. In this case, statement (2)(ii) is valid.

Suppose $\Omega$ has two connected components. Then $x_{3}=0$ and the number of edges between $\Omega$ and $\Gamma-\Omega$ is equal to $18 \cdot 12$. Hence, $x_{1}+x_{2}=150$ and $x_{1}+2 x_{2}=216$; therefore, $x_{2}=66$ and $x_{1}=84$. Let $a \in \Omega$ and $[a]$ contain $\delta_{i}$ vertices from $X_{i}$. Then $\delta_{1}+\delta_{2}=18$, and the union of the $\mu$-subgraphs of $a$ with vertices in the second connected component contains 18 vertices from $X_{2}$. Thus $\delta_{2}=18$, a contradiction.

Now assume that $\alpha>0$ and a vertex $u$ is isolated in $\Omega$. For $y \in \Omega$, we denote $\left|[y] \cap X_{3}\right|$ by $\sigma_{y}$. If $\beta=2$ and $\Omega^{1}$ and $\Omega^{2}$ are distinct $K_{3,3}$-components of $\Omega$, then the union of the $\mu$-subgraphs of $u$ with vertices in the connected components of the graph $\Omega-\{u\}$ contains 21 vertices from $X_{3}$. Therefore, $\alpha=1$ and $X_{0}\left(\Omega^{1} \cup \Omega^{2}\right)$ contains the $K_{3,3}$-subgraph $\left\{u=u_{1}, u_{2}, u_{3} ; a_{1}, a_{2}, a_{3}\right\}$, where $u_{i} \in \Omega$ and $a_{i} \in X_{3}$. Hence, $\sum x_{i}=147$, $\sum i x_{i}=279$, and $x_{1}=0$. Thus, $x_{0}=36, x_{2}=54$, and $x_{3}=57$. In this case statement (2)(iii) holds true.

If $\beta=1$ and $\Omega^{1}$ is a unique $K_{3,3}$-component of the graph $\Omega$, then the number of 2 -paths with the initial vertex $u$ and the terminal vertex in $\Omega-\{u\}$ is equal to $3(3 \alpha+5)$. Therefore, $\alpha \leq 3$, and for $\alpha=3$ the subgraph $[u]$ is contained in $X_{3}$. In this case, $X_{0}\left(\Omega^{1}\right)$ contains the $K_{3,3}$-subgraph $\left\{u=u_{1}, u_{2}, u_{3} ; a_{1}, a_{2}, a_{3}\right\}$, where $u_{i} \in \Omega, a_{i} \in X_{3}$, and for $w \in \Omega^{1}$ the subgraph $[u] \cap[w]$ is contained in the neighborhood of a unique vertex in $\Omega-\left\{u_{1}, u_{2}, u_{3}\right\}$ isolated in $\Omega$; this contradicts the fact that $3 \alpha$ is odd. Let $\alpha=2$. Then $[u]$ contains $\sigma_{u}$ vertices from $X_{3}, 33-2 \sigma_{u}$ vertices from $X_{2}$, and $\sigma_{u}-12$ vertices from $X_{1}$. Therefore, $\sigma_{u}=12$ or $15, x_{0}+x_{1}+x_{2}+x_{3}=150$, and $x_{1}+2 x_{2}+3 x_{3}=234$. For $w \in \Omega^{1}$, the subgraph $[w]$ contains $\sigma_{w}$ vertices from $X_{3}, 3\left(6-2 \sigma_{w}\right)$ vertices from $X_{2}$, and $5 \sigma_{w}$ vertices from $X_{1}$. Therefore $\sigma_{w} \leq 3$. If the vertices in $\Omega-\Omega^{1}$ can be split into two triples lying in $K_{3,3}$-subgraphs that intersect $X_{3}$ at three vertices, then $x_{3} \leq 6+3 \cdot 6=24$. Since in this case $[u]$ contains at most 12 vertices from $X_{3}$, it follows that $x_{3}=24, \sigma_{u}=12$ for any vertex $u \in \Omega-\Omega^{1}$, and $\sigma_{w}=3$ for any vertex $w \in \Omega^{1}$. In this case, $x_{1}=90, x_{2}=27$, and $x_{0}=9$, which is impossible, because $x_{1}+2 x_{2}+3 x_{3}=90+54+72 \neq 234$. If $\Omega-\Omega^{1}$ contains no triple of vertices lying in a $K_{3,3}$-subgraph that intersects $X_{3}$ at three vertices, then $x_{3} \leq 18$, which contradicts the inequality $x_{3} \geq 6 \cdot 12 / 2$. If $\Omega-\Omega^{1}$ contains a unique 
triple of vertices that is contained in a $K_{3,3}$-subgraph intersecting $X_{3}$ at three vertices, then $x_{3} \leq 3+18=21$; as above, this contradicts the inequality $x_{3} \geq 3+(3 \cdot 9+3 \cdot 12) / 2$.

Let $\alpha=1$. Then $[u]$ contains $\sigma_{u}$ vertices from $X_{3}, 24-2 \sigma_{u}$ vertices from $X_{2}$, and $\sigma_{u}-3$ vertices from $X_{1}$. Therefore, $3 \leq \sigma_{u} \leq 12, x_{0}+x_{1}+x_{2}+x_{3}=153$, and $x_{1}+2 x_{2}+3 x_{3}=171$. For $w \in \Omega^{1}$, the subgraph $[w]$ contains $\sigma_{w}$ vertices from $X_{3}$, $3\left(3-2 \sigma_{w}\right)$ vertices from $X_{2}$, and $9+5 \sigma_{w}$ vertices from $X_{1}$. Hence, $\sigma_{w}=0$. If $\Omega-\Omega^{1}$ does not lie in a $K_{3,3}$-subgraph that intersects $X_{3}$ at three vertices, then $x_{3}=0$, which contradicts the fact that $3 \leq \sigma_{u}$ for $u \in \Omega-\Omega^{1}$. If the vertices of $\Omega-\Omega^{1}$ belong to a $K_{3,3}$-subgraph that intersects $X_{3}$ at three vertices, then $x_{3}=3, x_{2}=54, x_{1}=54$, and $x_{0}=42$, and statement (2)(iv) holds true. The lemma is proved.

We recall that a triple $(X, \mathcal{B}, I)$ is called a $(v, k, \lambda)$-design if $X$ is a set of points, $|X|=v, \mathcal{B}$ is a set of blocks, and $I$ is an incidence relation between the points and the blocks such that any block is incident to precisely $k$ points and any two points are incident to precisely $\lambda$ blocks. If $k=3$ and $\lambda=1$, the design is called a Steiner triple system.

Lemma 2.5. If $p=3$ and $\Omega$ is a $3 \alpha$-coclique, then $\alpha \leq 5$; moreover, if $\alpha=5$, the block design $\left(\Omega, X_{3}, I\right)$ is obtained by tripling the blocks of the Steiner triple system with $v=15$ and $r=7$.

Proof. Suppose $p=3$ and $\Omega$ is a $3 \alpha$-coclique. Then $\sum x_{i}=162-3 \alpha, \sum i x_{i}=63 \alpha$, and $x_{2}=3\left(\left(\begin{array}{c}3 \alpha \\ 2\end{array}\right)-3 x_{3}\right)$. If $u \in \Omega$ and $[u]$ contains $\sigma_{i}$ vertices from $X_{i}$, then $\sigma_{2}=$ $3\left(3 \alpha-1-2 \sigma_{3} / 3\right)$ and $\sigma_{1}=24-9 \alpha+\sigma_{3}$. Hence, $9 \alpha-24 \leq \sigma_{3} \leq(9 \alpha-3) / 2$ and $\alpha \leq 5$. Moreover, $\sigma_{i}$ is divisible by 3 . Let $\delta_{i}$ be the number of vertices in $\Omega$ with $\sigma_{3}=i$. If $\alpha=5$, then $\sigma_{3}=21$ and $\sigma_{2}=\sigma_{1}=0$. Next, $x_{3}=105, x_{1}=x_{2}=0$, and $x_{0}=42$. In this case, the block design $\left(\Omega, X_{3}, I\right)$, where the relation $I$ is induced by adjacency in the graph $\Gamma$, is obtained by tripling the blocks of the Steiner triple system with $v=15$ and $r=7$.

If $\alpha=4$, we have $12 \leq \sigma_{3} \leq 16.5$. If $\sigma_{3}=12$, then $\sigma_{2}=9$ and $\sigma_{1}=0$. If $\sigma_{3}=15$, then $\sigma_{2}=3$ and $\sigma_{1}=3$. Next, $x_{1}=3 \delta_{15}, x_{2}=\left(9 \delta_{12}+3 \delta_{15}\right) / 2$, and $x_{3}=\left(12 \delta_{12}+15 \delta_{15}\right) / 3$; therefore, $2\left(x_{1}+x_{2}+x_{3}\right)=2\left(150-x_{0}\right)=17 \delta_{12}+19 \delta_{15}$ and $48-x_{0}=\delta_{15}$.

For $\alpha=3$ we get $3 \leq \sigma_{3} \leq 12$. If $\sigma_{3}=3$, then $\sigma_{2}=18$ and $\sigma_{1}=0$. If $\sigma_{3}=6$, then $\sigma_{2}=12$ and $\sigma_{1}=3$. If $\sigma_{3}=9$, then $\sigma_{2}=6$ and $\sigma_{1}=6$. If $\sigma_{3}=12$, then $\sigma_{2}=0$ and $\sigma_{1}=9$. Next, $x_{1}=3 \delta_{6}+6 \delta_{9}+9 \delta_{12}, x_{2}=\left(18 \delta_{3}+12 \delta_{6}+6 \delta_{9}\right) / 2$, and $x_{3}=$ $\left(3 \delta_{3}+6 \delta_{6}+9 \delta_{9}+12 \delta_{12}\right) / 3$; therefore, $x_{1}+x_{2}+x_{3}=153-x_{0}=10 \delta_{3}+11 \delta_{6}+12 \delta_{9}+13 \delta_{12}$, and $63-x_{0}=\delta_{6}+2 \delta_{9}+3 \delta_{12}$.

For $\alpha=2$ we have $\sigma_{3} \leq 7.5$. If $\sigma_{3}=0$, then $\sigma_{2}=15$ and $\sigma_{1}=6$. If $\sigma_{3}=3$, then $\sigma_{2}=9$ and $\sigma_{1}=9$. If $\sigma_{3}=6$, then $\sigma_{2}=3$ and $\sigma_{1}=12$. Next, $x_{1}=6 \delta_{0}+9 \delta_{3}+12 \delta_{6}$, $x_{2}=\left(15 \delta_{0}+9 \delta_{3}+3 \delta_{6}\right) / 2$, and $x_{3}=\left(3 \delta_{3}+6 \delta_{6}\right) / 3$; therefore, $2\left(x_{1}+x_{2}+x_{3}\right)=2\left(156-x_{0}\right)=$ $27 \delta_{0}+29 \delta_{3}+31 \delta_{6}$ and $85-x_{0}=\delta_{3}+2 \delta_{6}$.

If $\alpha=1$, we have $\sigma_{3} \leq 3$. If $\sigma_{3}=0$, then $\sigma_{2}=6$ and $\sigma_{1}=15$. If $\sigma_{3}=3$, then $\sigma_{2}=0$ and $\sigma_{1}=18$. Next, $x_{1}=15 \delta_{0}+18 \delta_{3}, x_{2}=3 \delta_{0}$, and $x_{3}=\delta_{3}$; therefore, $x_{1}+x_{2}+x_{3}=159-x_{0}=18 \delta_{0}+19 \delta_{3}$ and $105-x_{0}=\delta_{3}$. If $\delta_{3}=0$, then $\delta_{0}=3$, $x_{1}=45, x_{2}=9$, and $x_{0}=105$. For $\delta_{3}=3$, we have $\delta_{0}=0, x_{1}=54, x_{2}=0, x_{3}=3$, and $x_{0}=102$. The lemma is proved.

\section{§3. Order 2 AUtomorphisms With FiXed POINTS}

In this section, we assume that $\Gamma$ is a strongly regular graph with parameters $(162,21$, $0,3)$ and that $t$ is an order 2 automorphism of the graph $\Gamma$ with a nonempty subgraph $\Omega=\operatorname{Fix}(t)$. Let $X_{i}$ be the set of vertices in $\Gamma-\Omega$ that are adjacent to precisely $i$ vertices in $\Omega, x_{i}=\left|X_{i}\right|$. 
Lemma 3.1. The following statements are valid:

(1) $x_{0}=\alpha_{1}(t), 3|\Omega|-x_{0}$ is divisible by 9 , and if $u \in X_{3}$ and $\left\{b_{1}, b_{2}, b_{3}\right\}=[u] \cap \Omega$, then $\left[b_{1}\right] \cap\left[b_{2}\right] \cap\left[b_{3}\right]$ contains $u, u^{t}$, and a vertex from $\Omega$;

(2) if $a$ vertex $b$ has degree 1 in the graph $\Omega$ and $a \in \Omega(b)$, then $\Omega \subset a^{\perp}$ and if $x_{0} \neq 0$, then $|\Omega| \leq 20$

(3) if $w \in X_{1}, w$ is adjacent to a vertex $e \in \Omega$ of degree $k^{\prime}$ in $\Omega$, and [w] contains $y_{i}$ vertices from $X_{i}$, then $k^{\prime}=3|\Omega|-63+3 y_{0}+2 y_{1}$ and $\Omega \leq 26$;

(4) $\Omega$ is neither a clique, nor a strongly regular graph.

Proof. Let $p=2$. As above, $x_{2}=0$. First, we note that $\left|X_{0}\right|=\alpha_{1}(t)$, because a vertex $u$ lies in $X_{0}$ if and only if $u$ and $u^{t}$ are adjacent. Next, the degree of every vertex in the graph $\Omega$ is odd and $\chi_{1}(t)=\left(3|\Omega|-\alpha_{1}(t)\right) / 9+2$; therefore, $3|\Omega|-x_{0}$ is divisible by 9 . Let $w \in X_{3}$, let $b_{1}, b_{2} \in[w] \cap \Omega$, and let $a$ be a unique vertex in $\Omega\left(b_{1}\right) \cap \Omega\left(b_{2}\right)$. Then $[a] \cap[w]$ contains three vertices from $\Omega$. Statement (1) is proved.

If a vertex $b$ has degree 1 in the graph $\Omega$ and $a \in \Omega(b)$, then for $c \in \Omega-\{a, b\}$, the subgraph $[b] \cap[c]$ contains a vertex from $\Omega$ and $\Omega \subset a^{\perp}$.

Suppose $|\Omega| \geq 20$ and $u \in X_{0}$. Then $[u]$ contains $u^{t}$, and the union of the $\mu$-subgraphs of the form $[u] \cap[a]$ with $a \in \Omega$ contains at least 20 vertices of $X_{3}$. Hence, $|\Omega|=20$ and $[u]-\left\{u^{t}\right\} \subset X_{3}$. Statement (2) is proved.

Let $w \in X_{1}$, and let $w$ be adjacent to a vertex $e \in \Omega$ of degree $k^{\prime}$ in $\Omega$. If $[w]$ contains $y_{i}$ vertices of $X_{i}$, then $y_{0}+y_{1}+y_{3}=20$, and the number of edges between $\Omega-\{e\}$ and $[w]-\{e\}$ is equal to $y_{1}+3 y_{3}=2 k^{\prime}+3\left(|\Omega|-1-k^{\prime}\right)$; therefore, $k^{\prime}=3|\Omega|-63+3 y_{0}+2 y_{1}$. If $|\Omega| \geq 28$, then $k^{\prime} \geq 21$, which contradicts the fact that $e$ is adjacent to $w$. Statement (3) is proved.

If $\Omega$ is a clique, then $|\Omega|=2$ and $\chi_{1}(t)=\left(6-\alpha_{1}(t)\right) / 9+2$. Therefore $\alpha_{1}(t)-6$ is divisible by 9 , in contradiction with $x_{0}=120$.

Suppose $\Omega$ is not a clique. Then $\Omega$ is a graph without triangles of diameter 2 in which $|\Omega(a) \cap \Omega(b)|$ equals 1 or 3 for any two nonadjacent vertices $a, b \in \Omega$.

If $\Omega$ is a strongly regular graph, then $\Omega$ is one of the Moore graphs or a $K_{3,3}$-subgraph. If $\Omega$ is a Petersen graph, then $x_{3}=20, x_{0}+x_{1}=132$, and $x_{1}+3 x_{3}=180$. Hence, $x_{0}=12$ and $x_{1}=120$. If $u \in X_{3}$, then [u] contains 12 vertices from $X_{1}$ and 6 vertices from $X_{0}$. Therefore the number of edges between $X_{3}$ and $X_{0}$ is equal to 120 . If $z \in X_{0}$, then $[z]$ contains 10 vertices from $X_{3}$ and 11 vertices from $X_{0}$; this contradicts the fact that $X_{0}$ is a 12-clique.

We show that $\Omega$ is not a Hoffman-Singleton graph. Otherwise, for $a \in \Omega$ every pair of vertices in $\Omega(a)$ belongs to a unique triple of vertices in $\Omega(a)$ that lie in the neighborhoods of two vertices $u$ and $u^{t}$. Hence, every vertex in $\Omega(a)$ is adjacent to six vertices in $X_{3}$ and eight vertices in $X_{1}$, which contradicts the fact that then $x_{1}=400$.

If $\Omega=K_{3,3}$, then $x_{2}=x_{3}=0$. Next, $x_{0}=48$ and $3|\Omega|-x_{0}$ is not divisible by 9 ; this contradicts statement (1).

Lemma 3.2. The following statements are valid:

(1) if $\Omega$ is contained in $a^{\perp}$, then $|\Omega|=4$ and $x_{1}=72, x_{3}=2$, and $x_{0}=84$;

(2) if $u \in X_{3},[u]$ contains $z_{i}$ vertices in $X_{i},\left\{b_{1}, b_{2}, b_{3}\right\}=[u] \cap \Omega$, and the degree of $b_{i}$ in $\Omega$ is equal to $\beta_{i}+1$, then $3|\Omega|-30+z_{0}-2 z_{3}=\beta_{1}+\beta_{2}+\beta_{3}$ and $z_{0}$ is even;

(3) the parameter $x_{1}$ is not equal to 0 ;

(4) $|\Omega| \leq 18$, and if $x_{0}=0$, then $|\Omega|=18$.

Proof. If $\Omega \subset a^{\perp}$, then every pair of vertices in $\Omega(a)$ lies in a unique triple of vertices in $\Omega(a)$ that occurs in the neighborhoods of two vertices from $X_{3}$. Hence, every vertex in $\Omega(a)$ is adjacent to $|\Omega|-2$ vertices from $X_{3}$ and to $22-|\Omega|$ vertices from $X_{1}$. Therefore, $x_{1}=|\Omega|(22-|\Omega|), x_{3}=(|\Omega|-1)(|\Omega|-2) / 3$, and $x_{0}=162-|\Omega|-|\Omega|(22-|\Omega|)-$ $(|\Omega|-1)(|\Omega|-2) / 3$. Since $\chi_{1}(t)=\left(3|\Omega|-x_{0}\right) / 9+2$, it follows that $|\Omega|^{2}+3|\Omega|-1$ 
is divisible by 27 . If $|\Omega|$ is congruent to $r$ modulo 9 , then $r^{2}+3 r-1$ is divisible by 9 , and thus $r=2$ or 4 . Hence, $|\Omega|$ is equal to 4,20 or 22 . If $|\Omega|=20$, then $x_{1}=40, x_{3}=114, x_{0}=-12$, a contradiction. If $|\Omega|=22$, then $x_{3}=140$ and $3|\Omega|-x_{0}$ is not divisible by 9 , a contradiction. Therefore, $|\Omega|=4$ and $x_{1}=72, x_{3}=2, x_{0}=84$. Statement (1) is proved.

Suppose $\Omega$ does not lie in $a^{\perp}$ for any vertex $a$. Then $\Omega$ contains two pairs of nonadjacent vertices $p, q$ and $r, s$ such that $[p] \cap[q] \subset \Omega$ and $[r] \cap[s]$ contains a unique vertex in $\Omega$; in particular, $x_{3} \neq 0$.

Suppose $u \in X_{3},[u]$ contains $z_{i}$ vertices from $X_{i},\left\{b_{1}, b_{2}, b_{3}\right\}=[u] \cap \Omega$, and the degree of $b_{i}$ in $\Omega$ is equal to $\beta_{i}+1$. Then $\beta_{i}$ is even, $z_{0}+z_{1}+z_{3}=18$, and the number of edges between $\Omega-[a]$ and $[a]-\Omega$ is equal to $z_{1}+3 z_{3}=2\left(\beta_{1}+\beta_{2}+\beta_{3}\right)+3\left(|\Omega|-\left(\beta_{1}+\beta_{2}+\beta_{3}\right)-4\right)$. Replacing $z_{1}+z_{3}$ by $18-z_{0}$, we obtain $3|\Omega|-30+z_{0}-2 z_{3}=\beta_{1}+\beta_{2}+\beta_{3}$. Since $\beta_{i}$ is even, so is $z_{0}$. Statement (2) is proved.

We show that $x_{1} \neq 0$. Suppose $x_{1}=0$. Then $\beta_{i} \geq 2, z_{3}=18-z_{0}$, and $3|\Omega|-66+3 z_{0}=$ $\beta_{1}+\beta_{2}+\beta_{3}$. Suppose $x_{0}=0$. Then $|\Omega|$ is divisible by 6 . If $|\Omega|=36$, then $\beta_{1}+\beta_{2}+\beta_{3}=42$. In this case, $X_{3}$ is a regular graph of degree 18 with 126 vertices, which contradicts Lemma 2. If $|\Omega|=24$, then $\beta_{1}+\beta_{2}+\beta_{3}=6$. In this case, $\Omega$ is a regular graph of degree 3 ; this contradicts the fact that then the diameter of $\Omega$ is greater than 2 . Therefore, $|\Omega|=30$ and $\beta_{1}+\beta_{2}+\beta_{3}=24$. In this case, $X_{3}$ is a regular graph of degree 18 with 132 vertices, which contradicts Lemma 2. Now $x_{0} \neq 0$ and $|\Omega| \leq 20$. Since a vertex in $\Omega$ is adjacent to $x_{0}$ vertices in $X_{3}$, it follows that $x_{0} \leq 18, z_{0} \leq 9$, and $|\Omega| \geq 16$. If $|\Omega|=16$, then $x_{0}-3$ is divisible by 9 and $x_{0}=12$; this contradicts the fact that $z_{0}-6=\left(\beta_{1}+\beta_{2}+\beta_{3}\right) / 3$. If $|\Omega|=18$, then $x_{0}$ is divisible by 9 and $x_{0}=18$. In this case, $\Omega$ is a regular graph of degree 3 , and the diameter of $\Omega$ is greater than 2 , a contradiction. If $|\Omega|=20$, then $x_{0}+3$ is divisible by 9 and $x_{0}=6$, which is impossible because $z_{0}-2=\left(\beta_{1}+\beta_{2}+\beta_{3}\right) / 3$. Thus, $x_{1} \neq 0$. Statement $(3)$ is proved.

Let $x_{0}=0$. If $|\Omega|=12$, then $6-2 z_{3}=\beta_{1}+\beta_{2}+\beta_{3}$; therefore $\beta_{i}=2$ and $z_{3}=0$. Thus, $X_{3}$ is a coclique, and a vertex in $X_{3}$ is adjacent to 18 vertices in $X_{1}$. Let a vertex $u$ in $X_{3}$ be adjacent to vertices $b_{1}, b_{2}, b_{3} \in \Omega$. By Lemma 3.1, $\left[b_{1}\right] \cap\left[b_{2}\right] \cap\left[b_{3}\right]$ contains $u, u^{t}$, and a vertex $a$ in $\Omega$. Since $\beta_{i}=2$, the degree of $a$ in $\Omega$ is equal to 5 . The vertex $a$ is not adjacent to any vertices from $X_{3}$, and thus, every vertex in $\Omega_{2}(a)$ is adjacent to three vertices in $\Omega(a)$, and the number of edges between $\Omega_{2}(a)$ and $\Omega(a)$ is equal to 18. On the other hand, $\Omega(a)$ contains three vertices $b_{i}$ each of which is adjacent to two vertices in $\Omega_{2}(a)$; this contradicts the fact that each of the two remaining vertices in $\Omega(a)$ is adjacent to six vertices in $\Omega_{2}(a)$.

Let $|\Omega|=24$. If $w \in X_{1}$, then $[w] \cap\left[w^{t}\right]$ contains a vertex $e \in \Omega$ and vertices $u, u^{t} \in X_{1}$. Next, $[u] \cap[e]$ contains $w, w^{t}$, and a vertex $d \in \Omega$. Let $e$ have degree $k^{\prime}$ in $\Omega$, and let $[w]$ contain $y_{i}$ vertices from $X_{i}$. By Lemma 6 , we have $k^{\prime}=9+2 y_{1}$. Since $y_{1} \geq 2$, it follows that the degrees of the vertices $d$ and $e$ in the graph $\Omega$ are at least 13, which is impossible because then $|\Omega| \geq 26$. Thus, if $x_{0}=0$, then $|\Omega|=18$.

Suppose $|\Omega| \geq 20$. Then there is a vertex $u \in X_{0}$. But $[u]$ contains $u^{t}$, and the union of the $\mu$-subgraphs of the form $[u] \cap[a]$, where $a \in \Omega$, contains at least 20 vertices from $X_{3}$. Hence, $|\Omega|=20$ and $[u]-\left\{u^{t}\right\} \subset X_{3}$. Since $\chi_{1}(t)=\left(3|\Omega|-x_{0}\right) / 9+2$, we see that $x_{0}$ is 6,24 , or 42 . In the last case, by Lemma 2 , every vertex off $X_{0}$ is adjacent to seven vertices from $X_{0}$, which contradicts the fact that no two vertices in $X_{1}$ in $X_{0}$ are adjacent. If $d \in \Omega$ and $[d]$ contains $2 r$ vertices from $X_{3}$, then $\Omega_{2}(d)$ includes pairs of vertices adjacent to pairs of vertices from $[d] \cap X_{3}$ and $r \leq 8$. Next, $3 \leq \Omega(d) \leq 19-2 r$ and $[d]$ contains at least two vertices from $X_{1}$; therefore, $x_{1} \geq 40$.

Let $\left\{u_{1}, u_{1}^{t}\right\},\left\{u_{2}, u_{2}^{t}\right\}$, and $\left\{u_{3}, u_{3}^{t}\right\}$ be pairwise distinct edges from $X_{0}$. Then $X_{3}$ contains 40 vertices from $\left[u_{1}\right] \cup\left[u_{1}^{t}\right], 28$ vertices from $\left(\left[u_{2}\right]-\left(\left[u_{1}\right] \cup\left[u_{1}^{t}\right]\right) \cup\left(\left[u_{2}^{t}\right]-\left(\left[u_{1}\right] \cup\left[u_{1}^{t}\right]\right)\right.\right.$, 
and at least 16 vertices from $\left(\left[u_{3}\right]-\left(\left[u_{1}\right] \cup\left[u_{1}^{t}\right] \cup\left[u_{2}\right] \cup\left[u_{2}^{t}\right]\right)\right) \cup\left(\left[u_{3}^{t}\right]-\left(\left[u_{1}\right] \cup\left[u_{1}^{t}\right] \cup\left[u_{2}\right] \cup\left[u_{2}^{t}\right]\right)\right)$; thus, $x_{3} \geq 84$. If $x_{0}=24$, then $x_{1}+x_{3}=118$ and $x_{1} \leq 34$, a contradiction. If $x_{0}=6$, then a certain vertex in $X_{3}$ is adjacent with a unique vertex in $X_{0}$, which contradicts Lemma 2.2. Thus, $|\Omega| \leq 18$. The lemma is proved.

Lemma 3.3. Let $\Delta=\left\{a_{1}, a_{2}, a_{3} ; b_{1}, b_{2}, b_{3}\right\}$ be a $K_{3,3}$-subgraph in $\Omega$. Then the following statements hold true:

(1) $\left|\Omega \cap X_{0}(\Delta)\right|=0$;

(2) if a vertex $c$ in $\Omega\left(a_{1}\right)-\Delta$ is adjacent to two vertices $d_{1}$ and $d_{2}$ in $\Omega\left(b_{1}\right)$, then $\Omega\left(a_{1}\right)-\Delta$ contains another vertex adjacent to $d_{1}$ and $d_{2}$;

(3) if $\Omega\left(a_{1}\right)-\Delta$ contains a vertex $c$, then $\left[a_{1}\right] \cap\left[p_{i}\right] \cap\left[p_{i}^{t}\right]=\left\{b_{i}, c, c_{i}\right\}$ for some vertices $c_{i} \in \Omega$ and $p_{i} \in X_{3} \cap\left[b_{i}\right]$, for $e \in\left[a_{2}\right] \cap \Omega(c)$ we have $\left[a_{2}\right] \cap\left[q_{i}\right] \cap\left[q_{i}^{t}\right]=\left\{b_{i}, e, e_{i}\right\}$ for some vertices $e_{i} \in \Omega$ and $q_{i} \in X_{3} \cap\left[b_{i}\right]$, and for $f \in\left[a_{3}\right] \cap \Omega(c)$ we obtain $\left[a_{3}\right] \cap\left[r_{i}\right] \cap\left[r_{i}^{t}\right]=$ $\left\{b_{i}, f, f_{i}\right\}$ for some vertices $f_{i} \in \Omega$ and $r_{i} \in X_{3} \cap\left[b_{i}\right]$; the degrees of the vertices $b_{i}$ in $\Omega$ are equal to 3 , and $|\Omega|=18$.

Proof. Let $c \in \Omega \cap X_{0}(\Delta)$. The $\mu$-subgraphs of the vertex $c$ and of vertices in $\Delta$ contain at least six vertices in $\Omega$. Since the degree of the vertex $c$ in $X_{0}$ is equal to 3 , it follows that $X_{0}(c)$ contains a vertex $d$ in $\Omega$ and $|\Omega| \geq 20$, a contradiction. Statement (1) is proved.

If $[c] \cap\left[b_{1}\right]$ contains two vertices $d_{1}$ and $d_{2}$ in $\Omega-\Delta$, but $\Omega\left(a_{1}\right)-\left\{b_{1}, c\right\}$ contains no vertices adjacent to $d_{1}$ and $d_{2}$, then $\left[d_{i}\right] \cap\left[a_{1}\right]$ contains two vertices of $\Omega-\Delta$ for $i=1,2$ and the degrees of the vertices $a_{1}$ and $b_{1}$ in $\Omega$ are at least 7 . Since either of the subgraphs $\left[a_{i}\right] \cap[c]$ and $\left[b_{i}\right] \cap\left[d_{1}\right]$ contains one vertex in $\Omega-\Delta$, it follows that the degrees of the vertices $a_{i}$ and $b_{i}$ in $\Omega$ are at least 5 for $i=1,2$; this is impossible because $|\Omega| \geq 20$. Statement (2) is proved.

We assume that $\Omega\left(a_{1}\right)-\Delta$ contains the vertex $c$ and we show that $[c] \cap \Omega\left(b_{i}\right)=\left\{a_{1}\right\}$ for $i \in\{1,2,3\}$. Otherwise, by statement (1), we may assume that $\Omega\left(a_{1}\right)-\Delta$ contains vertices $c$ and $c^{\prime}$ adjacent to vertices $d$ and $d^{\prime}$ in $\Omega\left(b_{1}\right)-\Delta$. Next, $[c] \cap\left[a_{i}\right]$ contains a vertex $c_{i}$ in $\Omega-\Delta$, and $\left[c^{\prime}\right] \cap\left[a_{i}\right]$ contains a vertex $c_{i}^{\prime}$ in $\Omega-\Delta$ for $i=2,3$. Symmetrically, $[d] \cap\left[b_{i}\right]$ contains a vertex $d_{i}$ in $\Omega-\Delta$, and $\left[d^{\prime}\right] \cap\left[b_{i}\right]$ has a vertex $d_{i}^{\prime}$ in $\Omega-\Delta$ for $i=2,3$. Hence, $|\Omega|=18$. If $c_{2}$ and $d_{2}$ are not adjacent, then $\left[d_{2}\right] \cap\left[c_{2}\right]$ has a vertex $e$ in $\Omega-\Delta$. Clearly, $e \in\left\{c_{3}, c_{3}^{\prime}, d_{3}, d_{3}^{\prime}\right\}$. Without loss of generality, $e=c_{3}$. Then the vertices $c_{3}$ and $c_{3}^{\prime}$ are adjacent to the vertices $d_{2}$ and $d_{2}^{\prime}$. Thus, renumbering the vertices $d_{i}$ and $d_{i}^{\prime}$, we may assume that $c_{2}$ and $c_{2}^{\prime}$ are adjacent to $d_{2}$ and $d_{2}^{\prime}$. Next, $c_{3} \in[c] \cap\left[c_{2}^{\prime}\right], d_{3} \in[d] \cap\left[d_{2}^{\prime}\right]$, and the vertices $c_{3}$ and $c_{3}^{\prime}$ are adjacent to the vertices $d_{3}$ and $d_{3}^{\prime}$, which contradicts the fact that $\left[c_{3}\right] \cap\left[d_{2}\right]$ contains precisely two vertices $c_{2}^{\prime}$ and $d_{3}^{\prime}$ in $\Omega$.

Thus, for $c \in \Omega\left(a_{1}\right)-\Delta$ we have $[c] \cap \Omega\left(b_{i}\right)=\left\{a_{1}\right\}$ for $i \in\{1,2,3\}$. Then $[c] \cap\left[b_{i}\right]=$ $\left\{a_{1}, p_{i}, p_{i}^{t}\right\}$ for a vertex $p_{i} \in X_{3}$. By Lemma 3.1, $\left[a_{1}\right] \cap\left[p_{i}\right] \cap\left[p_{i}^{t}\right]=\left\{b_{i}, c, c_{i}\right\}$ for some vertex $c_{i}$ in $\Omega$. Similarly, if $e \in\left[a_{2}\right] \cap \Omega(c)$, then there are vertices $q_{i} \in X_{3}$ such that $\left[a_{2}\right] \cap\left[q_{i}\right] \cap\left[q_{i}^{t}\right]=\left\{b_{i}, e, e_{i}\right\}$ for some vertex $e_{i}$ in $\Omega$. Finally, for $f \in\left[a_{3}\right] \cap \Omega(c)$ there are vertices $r_{i} \in X_{3}$ such that $\left[a_{3}\right] \cap\left[r_{i}\right] \cap\left[r_{i}^{t}\right]=\left\{b_{i}, f, f_{i}\right\}$ for some vertex $f_{i}$ in $\Omega$. Thus, the degrees of the vertices $a_{i}$ in $\Omega$ are equal to 7 ; therefore, the degrees of the vertices $b_{i}$ in $\Omega$ are equal to 3 and $|\Omega|=18$.

Lemma 3.4. $\Omega$ contains no $K_{3,3}$-subgraphs.

Proof. Let $\Delta=\left\{a_{1}, a_{2}, a_{3} ; b_{1}, b_{2}, b_{3}\right\}$ be a $K_{3,3}$-subgraph of $\Omega$. Since $\Omega \neq \Delta$, statement (1) of Lemma 3.4 allows us to assume that $\Omega\left(a_{1}\right)-\Delta$ has a vertex $c$. By statement (3) of Lemma 3.3, we have $\left[a_{1}\right] \cap \Omega=\left\{c, c_{1}, c_{2}, c_{3}\right\},\left[a_{2}\right] \cap \Omega=\left\{e, e_{1}, e_{2}, e_{3}\right\}$, and $\left[a_{3}\right] \cap \Omega=$ $\left\{f, f_{1}, f_{2}, f_{3}\right\}$. If $e$ is adjacent to $c_{1}, c_{2}$, and $c_{3}$, then either $c$ is adjacent to $e_{1}, e_{2}$, and $e_{3}$, or a certain vertex $e_{i}$ is adjacent to a vertex in $\left\{c_{1}, c_{2}, c_{3}\right\}$. In the latter case we may assume that $c_{1}$ is adjacent to $e, e_{1}$, and $e_{2}$. 
The subgraph $\left[c_{1}\right] \cap\left[c_{2}\right]$ contains $a_{1}, c$, and yet another vertex in $\Omega$. For this reason, we may assume that either $f$ is adjacent to $c_{1}, c_{2}$, and $c_{3}$, or the pairs of vertices $c_{i}, c_{j}$ are adjacent to $f_{6-i-j}$ for distinct $i, j \in\{1,2,3\}$. Let $\left\{a_{1}, e, f ; c_{1}, c_{2}, c_{3}\right\}$ be a $K_{3,3}$-subgraph. If $c$ is adjacent to $e_{1}, e_{2}, e_{3}$, then the vertex $e_{i}$ is not adjacent to any of the vertices from $\left\{c_{1}, c_{2}, c_{3}\right\}$; therefore, the vertices $e_{1}, e_{2}, e_{3}$ are adjacent to $f$ and the vertices $f_{1}, f_{2}, f_{3}$ are adjacent to $e$ and $c$. In this case, $\left[b_{1}\right] \cap[c]$ contains $a_{1}, p$, and $p^{t}$ for a vertex $p$ from $X_{3}$, and $\left[a_{1}\right] \cap[p] \cap\left[p^{t}\right]=\left\{b_{1}, c, c_{i}\right\}$ for some $i \in\{1,2,3\}$. For $j \neq i$, the subgraph $\left[b_{1}\right] \cap\left[c_{j}\right]$ contains $a_{1}, q$, and $q^{t}$ for a vertex $q$ from $X_{3}$, and $\left[a_{1}\right] \cap[q] \cap\left[q^{t}\right]=\left\{b_{1}, c, y\right\}$ for $y \in\left\{b_{1}, b_{2}, b_{3}\right\} \cup\left\{c_{1}, c_{2}, c_{3}\right\}$; this contradicts the fact that a vertex outside a $K_{3,3}$-subgraph is adjacent to at most one of its vertices.

If $c_{1}$ is adjacent to $e, e_{1}$, and $e_{2}$, then $c$ and $f$ are adjacent to $e_{3}$ and each of the subgraphs $\left[e_{1}\right] \cap\left[c_{2}\right]$ and $\left[e_{1}\right] \cap\left[c_{3}\right]$ contains a vertex from $\left\{f_{1}, f_{2}, f_{3}\right\}$. Similarly, each of the subgraphs $\left[e_{2}\right] \cap\left[c_{2}\right]$ and $\left[e_{2}\right] \cap\left[c_{3}\right]$ contains a vertex from $\left\{f_{1}, f_{2}, f_{3}\right\}$, which contradicts the fact that then the vertices $\left\{f_{1}, f_{2}, f_{3}\right\}$ are adjacent to $e_{1}$ and $e_{2}$.

Suppose that the pairs of vertices $c_{i}, c_{j}$ are adjacent to $f_{6-i-j}$ for distinct $i, j \in$ $\{1,2,3\}$. Then the vertices $f_{1}, f_{2}$, and $f_{3}$ are adjacent to $c$ and the vertices $c_{1}, c_{2}$, and $c_{3}$ are adjacent to $f$. If $c_{1}$ is adjacent to $e, e_{1}$, and $e_{2}$, then $f_{2}$ and $f_{3}$ are adjacent to $a_{3}, c, c_{1}$, and $e_{3}$, a contradiction. Therefore, $\left[a_{2}\right] \cap\left[c_{i}\right]$ contains a unique vertex $e$ in $\Omega$, so that $e_{1}, e_{2}$, and $e_{3}$ are adjacent to $c$ and the vertices $f_{1}, f_{2}$, and $f_{3}$ are adjacent to $e$; this contradicts the fact that $\left[f_{1}\right] \cap\left[f_{2}\right]$ contains $a_{3}, c, e$, and $c_{3}$.

Lemma 3.5. $\Omega$ is a 3-paw.

Proof. Suppose $\Omega$ is not a 3 -paw. Then there are vertices $a, b \in \Omega$ such that $[a] \cap[b]$ contains three vertices $c_{1}, c_{2}$, and $c_{3}$ in $\Omega$. By Lemma 3.4, the subgraph $\left[c_{i}\right] \cap\left[c_{j}\right]$ contains three vertices $a, b$, and $d_{6-i-j}$ in $\Omega$ for any distinct $i, j \in\{1,2,3\}$. Next, $[a] \cap\left[d_{1}\right]$ contains three vertices $e_{1}, c_{2}$, and $c_{3}$ in $\Omega$. Similarly, the vertex $d_{i}$ is adjacent to a vertex $e_{i} \in[a]-\left\{c_{1}, c_{2}, c_{3}\right\}$ for $i=2,3$. Symmetrically, the vertex $d_{i}$ is adjacent to a vertex $f_{i} \in[b]-\left\{c_{1}, c_{2}, c_{3}\right\}$. Since the degrees of vertices in $\Omega$ are odd, it follows that $\Omega(a)$ additionally contains a vertex $a^{\prime}, \Omega(b)$ contains a vertex $b^{\prime}$, and we find 16 vertices in $\Omega$; this contradicts the fact that $\left[c_{i}\right] \cap\left[d_{i}\right]$ contains a new vertex in $\Omega$ for $i=1,2,3$. The lemma and, thus, the theorem are proved.

\section{REFERENCES}

[1] A. A. Makhnev and D. V. Paduchikh, On automorphisms of the Aschbacher graph, Algebra Logika 40 (2001), no. 2, 125-134; English transl., Algebra Logic 40 (2001), no. 2, 69-74. MR1850442 (2002f:20002)

[2] A. A. Makhnev and V. V. Nosov, On automorphisms of strongly regular graphs with $\lambda=0, \mu=$ 2, Mat. Sb. 195 (2004), no. 3, 47-68; English transl., Sb. Math. 195 (2004), nos. 3-4, 347-367. MR.2068957 (2005c:05200)

[3] A. E. Brouwer and W. H. Haemers, The Gewirtz graph: an exercise in the theory of graph spectra, European J. Combin. 14 (1993), 397-407. MR.1241907 (94k:05219)

[4] A. E. Brouwer, A. Cohen, and A. Neumaier, Distance-regular graphs, Ergeb. Math. Grenzgeb. (3), Bd. 18, Springer-Verlag, Berlin, 1989. MR.1002568 (90e:05001)

[5] P. Cameron, Permutation groups, London Math. Soc. Stud. Texts, vol. 45, Cambridge Univ. Press, Cambridge, 1999. MR1721031(2001c:20008)

Institute of Mathematics and Mechanics, Urals Branch, Russian Academy of Sciences, EkATERINBURG, Russia

E-mail address: puncker1978@mail.ru

Institute of Mathematics and Mechanics, Urals Branch, Russian Academy of Sciences, EKATERINBURG, RUSSIA

Received 9/DEC/2008

Translated by N. B. LEBEDINSKAYA 